\title{
Simuladores de compuertas lógicas para el aprendizaje de lógica proposicional en estudiantes de bachillerato
}

\section{Logic gate simulators for learning propositional logic in high school students}

\author{
María Belén Ordóñez-Parra \\ maria.ordonez.09@est.ucacue.edu.ec \\ Universidad Católica de Cuenca, Cuenca \\ Ecuador \\ https://orcid.org/0000-0001-5386-8979 \\ Darwin Gabriel García-Herrera \\ dggarciah@ucacue.edu.ec \\ Universidad Católica de Cuenca, Azogues \\ Ecuador \\ https://orcid.org/0000-0001-6813-8100 \\ Sandra Elizabeth Mena-Clerque \\ sandramena@ucacue.edu.ec \\ Universidad Católica de Cuenca, Cuenca \\ Ecuador \\ https://orcid.org/0000-0002-9186-2161
}

Recepción: 10 de agosto 2021

Revisado: 15 de septiembre 2021

Aprobación: 15 de noviembre 2021

Publicación: 01 de diciembre 2021 


\title{
RESUMEN
}

Una de las grandes dificultades de los estudiantes con la matemática es que intentan solo replicar procesos mecánicos y algoritmos abstractos, cuando se debe desarrollar un pensamiento crítico, analítico, creativo, razonamiento lógico y numérico que le ayude a desenvolverse en la sociedad del conocimiento. Por ello, este estudio analiza el impacto de simuladores de compuertas lógicas para el aprendizaje de lógica proposicional, con una metodología de tipo explicativa, con enfoque mixto entre los contenidos teóricos y la relación con la base de la electrónica digital. Con ayuda de una entrevista a un profesional en la rama de la matemática se planteará una estrategia que relacione la teoría con la práctica y mediante la observación a estudiantes de primero de bachillerato, se demostrará la motivación y comprensión de contenidos matemáticos utilizando simuladores de compuertas lógicas. De esta forma, se evidenciará que involucrarlos activamente en el aprendizaje aumenta la motivación y comprensión.

Descriptores: Lógica matemática; teoría de los conjuntos; pensamiento crítico. (Palabras tomadas del Tesauro UNESCO).

\begin{abstract}
One of the great difficulties of students with mathematics is that they only try to replicate mechanical processes and abstract algorithms, when they must develop critical, analytical, creative thinking, logical and numerical reasoning that helps them to function in the knowledge society. Therefore, this study analyzes the impact of logic gate simulators for the learning of propositional logic, with an explanatory methodology, with a mixed approach between the theoretical contents and the relationship with the basis of digital electronics. With the help of an interview with a professional in the field of mathematics, a strategy that relates theory to practice will be proposed and through observation of first year high school students, motivation and understanding of mathematical content will be demonstrated using logic gate simulators. In this way, it will be evident that actively involving them in learning increases motivation and understanding.
\end{abstract}

Descriptors: Mathematical logic; set theory; critical thinking. (Words taken from the UNESCO Thesaurus). 


\section{INTRODUCCIÓN}

Una dificultad presente en los jóvenes de Ecuador que intentan acceder a la educación superior es el bajo nivel de razonamiento verbal, lógico matemático y abstracto, esto se puede evidenciar en informes publicados por el INEVAL (2020), dónde el puntaje más bajo se da en la sección de razonamiento lógico-númerico, a pesar que la evalucación se centra en la comprensión lectora, el análisis, la relación y extración de información relevante, la toma de decisiones y resolución de problemas, más no en la memorización de contenidos teóricos o sólo en la ejecución de algoritmos complejos o procesos numéricos para resolver operaciones.

Se debe agregar que, el Ministerio de Educación (2016) en el currículo para matemática, plantea aprendizajes básicos necesarios para que los estudiantes puedan desenvolverse de manera autónoma e independiente en la sociedad, aplicando en su vida las competencias adquiridas durante su instrucción formal, por ello, el aprendizaje de la lógica proposicional utiliza textos con un valor de verdad y ayuda a mejorar la comprensión, así como la toma de desiciones mediante un proceso de razonamiento deductivo.

Por otro lado, la (UNESCO, 2013), hace referencia a cómo los jóvenes actuales nacieron en una cultura tecnológica, donde receptan información todo el tiempo, aquí surge la pregunta para los sistemas educativos actuales ¿están preparándoles con competencias para la sociedad del conocimiento?. El acceso y uso adecuado de las TIC permite fortalecer habilidades y destrezas, además de, la capacidad para buscar y analizar información, un pensamiento crítico y razonamiento lógico que les ayude a responder a las demandas de la sociedad actual.

Tomando en cuenta lo anterior, las instituciones educativas, tratan de formar personas con competencias y creativas, que interactuén con la realidad y respondan a las necesidades de la sociedad actual, entonces, al direccionar el uso de las TIC, en este caso la aplicación Logic Circuit Simulator Pro para los procesos de aprendizaje de lógica proposicional en los estudiantes de primero de bachillerato, se busca fortalecer 
la comprensión, conocimientos, habilidades y destrezas que ayudan a mejorar el rendimiento académico y aplicar lo aprendido en la vida diaria.

\section{Referencial teórico}

Algunas dificultades presentes para que el rendimiento académico de los estudiantes sea bajo, se presenta en el estudio de realizado por (González-Vidal, 2020), en España, que el bajo rendimiento de los estudiantes se debe a causas como, ser parte de los grupos vulnerables, el poco o nulo acceso a internet, o el uso desmedido del mismo. Además, se menciona que para mejorar el rendimiento académico y fortalecer las competencias de los estudiantes, se debe realizar un control adecuado de acceso y uso de las TIC, con estrategias, actividades y tiempos planificados.

También, los aportes de (Rodríguez-Gómez et al. 2018), en España, tienen coincidencias con referencia al uso de las TIC en los estudiantes, señalan que está presente el uso problemático afectando al contexto personal y escolar de los jóvenes. En su población de estudio, se encontró que mientras más tiempo de acceso a dispositivos móviles e internet tienen los estudiantes, mejora sus competencias digitales, pero también, existen más problemas en los contextos antes mencionados. Por esta razón, el uso de las TIC debe ser planificado y las instituciones educativas deben impulsar el uso correcto y responsable de dispositivos tecnológicos.

Por otro lado, con respecto a la competencia matemática de los estudiantes, en el estudio realizado por (Mercader-Ruiz et al. 2017), también en España, se demuestra que la adquisición de las habilidades matemáticas básicas influye positivamente en el rendimiento académico posterior de la asignatura, debido a que, la mayoría de los contenidos están encadenados y existen habilidades que requieren de otras previas. Asimismo, en este estudio se sugiere que la adquisición de las habilidades y competencias matemáticas están en función de las actividades propuestas.

En cuanto a la relación entre contenidos académicos y las TIC, surge un tema interesante como lo es la robótica educativa, dónde se evidencia la motivación e interés de los estudiantes en el aprendizaje, siempre que las estrategias estén debidamente planeadas considerando el nivel académico y cognitivo del grupo, además de un acompañamiento continuo por parte de los docentes. Además, la 
preparación del docente fortalece la retroalimentación y elaboración de nuevas estrategias acorde a las dificultades de cada estudiante, personalizando el aprendizaje (Lopez-Caudana et al., 2020).

En cuanto a nuevas prácticas docentes, (Vega-Vega et al. 2015), en Colombia, mencionan la necesidad de innovar en las aulas, cambiar de paradigma tradicional donde el estudiante solo recibe información a uno donde ellos estén más involucrados con su entorno y puedan desarrollarse en un ambiente tecnológico, es decir, los contenidos se conviertan en algo práctico, que se pueda notar la relación con la vida diaria, además, que sea interesante para el alumnado. Las nuevas estrategias deben ser planificadas y evaluadas, aparte deben considerar todos los momentos del proceso enseñanza-aprendizaje.

Además del profesionalismo de los docentes, se debe agregar que la relación afectiva entre los docentes y estudiantes impacta favorablemente en la adquisición de habilidades matemáticas, en el estudio realizado por de (Zumaeta-Arista et al. 2018), en Perú, se recalca que la preocupación que muestra el docente hacia el contexto de sus alumnos, sus dificultades y un acompañamiento continuo con estrategias para el aprendizaje de cada uno, permite que los estudiantes estén más cómodos y dispuestos en la clase, favoreciendo la comprensión y ejecución de procesos propios de la asignatura.

Partiendo del uso de las TIC en la educación Ecuatoriana, el estudio de (Coronel-Maji et al. 2018), realizado a estudiantes de primer semestre de una carrera universitaria, señalan que el iniciar un tema incorporando una herramienta digital adecuada para el aprendizaje de los contenidos, tiene sus dificultades, pero con la práctica y la familiarización con las diversas herramientas, mediante las evaluaciones se demuestra que el nivel de comprensión del tema propuesto mejora notablemente.

Cómo se menciona anteriormente, la didáctica de la matemática implica la relación teórico-práctica de los contenidos, por eso en el estudio de (Rubio et al. 2020), en Ecuador, se indica que la articulación de los contenidos con las TIC, en su caso, con la programación y robótica, motiva a los estudiantes y mejora la comprensión de la lógica proposicional. Existen algunas dificultades con las actividades grupales, pero a 
pesar de ello, en la mayor parte de los estudiantes, el uso de las TIC si fortalece la adquisición de habilidades matemáticas.

\section{Lógica proposicional hacia circuitos lógicos}

La lógica proposicional es aquella que analiza la validez de las premisas que forman un enunciado y de esta manera dar una conclusión que también sea válida. Las premisas o proposiciones son aquellas oraciones a las que se les puede asignar un valor de verdadero o falso, por ejemplo, la manzana es azul. Las proposiciones son representadas por letras minúsculas y pueden unirse utilizando los conectores de lógicos para formar una proposición compuesta. Cabe mencionar que, las interrogaciones, exclamaciones y afirmaciones no son proposiciones (Aranda-Utrero, 2019).

Para conocer a los conectores lógicos y su expresión en lenguaje común, se empieza por el único operador que requiere de una proposición o resultado, la negación o no, el cuál cambia el valor de verdad de aquella proposición. Luego, están los operadores que requieren de dos o más proposiciones para poder aplicarse, se debe armar una tabla de verdad para analizar cada proposición de la expresión, entre ellos, la disyunción que en lenguaje común se dice o, con su resultado siendo falso sólo cuando ambas proposiciones son falsas, también se tiene a la conjunción, conocido como y que su conclusión es verdadera sólo cuando ambas proposiciones lo son.

El condicional conocido como el, si... entonces..., tiene un único caso de falsedad y es cuando la primera proposición o antecedente es verdadero y la segunda proposición o consecuente es falso, en los demás casos la conclusión es verdadera, mientras que, el bicondicional $\mathrm{o}, \ldots$ si y solo si..., es verdadero cuando las dos proposiciones tienen el mismo valor lógico, caso contrario es falso. Esto puede apreciarse en la tabla 1. 
Tabla 1.

Operadores lógicos.

\begin{tabular}{ccccccc}
\hline \multicolumn{2}{c}{ Proposiciones } & Negación & Disyunción & Conjunción & Condicional & Bicondicional \\
\hline $\mathrm{P}$ & $\mathrm{Q}$ & $\neg p$ & $p \vee q$ & $p \wedge q$ & $p \rightarrow q$ & $p \leftrightarrow q$ \\
$\mathrm{~V}$ & $\mathrm{~V}$ & $\mathrm{~F}$ & $\mathrm{~V}$ & $\mathrm{~V}$ & $\mathrm{~V}$ & $\mathrm{~V}$ \\
$\mathrm{~V}$ & $\mathrm{~F}$ & $\mathrm{~F}$ & $\mathrm{~V}$ & $\mathrm{~F}$ & $\mathrm{~F}$ & $\mathrm{~F}$ \\
$\mathrm{~F}$ & $\mathrm{~V}$ & $\mathrm{~V}$ & $\mathrm{~V}$ & $\mathrm{~F}$ & $\mathrm{~V}$ & $\mathrm{~F}$ \\
$\mathrm{~F}$ & $\mathrm{~F}$ & $\mathrm{~V}$ & $\mathrm{~F}$ & $\mathrm{~F}$ & $\mathrm{~V}$ & $\mathrm{~V}$ \\
\hline
\end{tabular}

Elaboración: Los autores.

Se debe agregar que (Carranza-Cortés, 2018), comenta que los operadores que son más intuitivos el momento de reconocerlos son la disyunción y la negación, mientras que la conjunción genera un poco de dificultades cuando se lo debe identificar dentro de proposiciones, esto se debe a que el aprendizaje de los operadores se relaciona más con memorizar una tabla y no en identificarlos de manera práctica, con situaciones que requieran un análisis y reflexión para proceder a la toma de decisiones.

Ahora, la lógica proposicional contribuye para el razonamiento y reflexión sobre cualquier situación real que se presente, por ello en el estudio de (Rincón-Rueda \& Ávila-Díaz, 2016), se menciona la relevancia del razonamiento lógico, crítico y el lenguaje computacional que juntos producen el pensamiento computacional, cómo todas las actividades tienden a ser ejecutadas o relacionadas con dispositivos tecnológicos actualmente, se debe comprender la lógica que universaliza el lenguaje computacional y así aprovechar los beneficios de estas herramientas en mejora de la sociedad.

Cuando se requiere facilidad y rapidez para procesar información, se utiliza de base al sistema numérico binario, donde los únicos dígitos que forman parte del mismo son $\{0,1\}$ y para comprenderlos mejor se pueden comparar con un interruptor, cuando el interruptor está apagado corresponde al cero en binario, mientras que al encender el 
interruptor permitiendo el paso de corriente en el circuito, esto equivale al uno (Lee \& Park, 2019).

En la actualidad, el álgebra de Boole es la base de los circuitos digitales, aquí los estados del circuito se pueden representar utilizando números binarios, debido a los dos estados que pueden presentarse en los terminales, encendido, activado o uno lógico y apagado, desactivado o cero. Hay tres compuertas lógicas AND, OR, NOT, las cuales comparten su tabla de verdad con la conjunción, disyunción y negación de la lógica proposicional respectivamente, además que el verdadero equivale a uno y el falso es cero, como se puede observar en la tabla 2.

Tabla 2.

Compuertas lógicas básicas.

\begin{tabular}{cccccc}
\hline Entrada $A$ & Entrada $B$ & OR $=\mathrm{A}+\mathrm{B}$ & $\mathrm{AND}=\mathrm{A}^{*} \mathrm{~B}$ & $\mathrm{NOT}=\overline{\boldsymbol{A}}$ & $\begin{array}{c}\text { NOT }= \\
\overline{\boldsymbol{B}}\end{array}$ \\
\hline 0 & 0 & 0 & 0 & 1 & 1 \\
0 & 1 & 1 & 0 & 1 & 0 \\
1 & 0 & 1 & 0 & 0 & 1 \\
1 & 1 & 1 & 1 & 0 & 0 \\
\hline
\end{tabular}

Elaboración: Los autores.

Los operadores lógicos de circuitos también tienen su tabla de verdad, se debe considerar que las entradas deben ser al menos dos en el caso de AND, OR y OR exclusivo, conocido como XOR, solo la compuerta NOT trabaja con una señal de entrada. Al igual que la lógica proposicional, si se requiere conocer todas las posibles combinaciones de estados lógicos en el circuito se aplica $2^{n}$, donde $\mathrm{n}$ representa la cantidad total de entradas (Li \& Xi, 2021).

También se utilizan algunas funciones lógicas que resultan de operar dos funciones básicas o más a la vez, éstas son XOR, NOR, NAND, XNOR, las cuales se presentan 
en la tabla 3 con sus respectivos valores lógicos de salida de acuerdo a las posibilidades lógicas de sus entradas.

\section{Tabla 3.}

Compuertas lógicas adicionales.

\begin{tabular}{cccccc}
\hline \multirow{2}{*}{ Entrada A } & \multirow{2}{*}{ Entrada B } & XOR & NAND & NOR & XNOR \\
\cline { 2 - 6 } & & $A \bar{B}+\bar{A} B$ & $\overline{A * B}$ & $\overline{A+B}$ & $\overline{A B}+A B$ \\
\hline 0 & 0 & 0 & 1 & 1 & 1 \\
0 & 1 & 1 & 1 & 0 & 0 \\
1 & 0 & 1 & 1 & 0 & 0 \\
1 & 1 & 0 & 0 & 0 & 1 \\
\hline
\end{tabular}

Elaboración: Los autores.

Es importante recalcar que las estrategias utilizadas para el aprendizaje de matemática dependen del docente y el contexto estudiantil, pero, para alcanzar los objetivos propuestos sobre un tema, el docente debe plantear actividades que conduzcan a los jóvenes a pensar más allá del proceso, sino, en la aplicación que le pueden dar al contenido en su vida diaria, además que, las actividades deben ser emocionantes o del interés del alumnado para que estén motivados a participar activamente de las sesiones o encuentros, con el acompañamiento adecuado de los docentes (Zhang et al. 2020).

Considerando el contenido, su utilidad con los sistemas digitales y las TIC, la aplicación Logic Circuit Simulator Pro, la cual se encuentra de manera gratuita para dispositivos móviles, permite crear circuitos lógicos para comprender como funciona la electrónica digital básica, además contiene una variedad de elementos lógicos como las compuertas, interruptores, leds. Además, se pueden realizar simulaciones de los circuitos para comprobar su funcionamiento, esto con fines educativos ( $\mathrm{Li} \& \mathrm{Xi}$, 2021). 
Con la versión libre, se puede acceder a tutoriales, además de contenido explicativo de cada elemento que puede utilizarse para formar un circuito lógico, así como las tablas de verdad de las compuertas. Las restricciones son poco perceptibles debido a que un estudiante de bachillerato está iniciando con simulaciones, por lo cual, no realizará circuitos complejos o con muchos elementos.

Entonces, no sólo se debe transmitir contenidos y procesos para la resolución de enunciados, sino, los estudiantes deben ser capaces de pensar, reflexionar, buscar estrategias para resolver una dificultad planteada o poder detectar y plantear una dificultad para alcanzar solventarla, esto se logra sólo con la relación de los estudiantes con el medio que los rodea, es decir, los contenidos deben reflejar claramente la relación teórico-práctica, además de que en la práctica debe ser ingenioso, llamativo y cercano a la realidad del estudiante y así se alcanzará a cautivar su atención.

\section{MÉTODO}

El presente estudio de investigación fue de tipo experimental que según (HernándezSampieri et al. 2014), existe una relación causa - efecto entre la variable independiente, uso de Logic Circuit Simulator Pro y la variable dependiente, aprendizaje de lógica proposicional, además de una metodología mixta, de ésta forma se obtuvo más información y de distintas fuentes que ayudaron a contrastar los datos teóricos y los numéricos correspondientes a la relación existente entre las variables del objeto de estudio, para ello la recolección de información fue de tipo transversal. Los datos fueron recolectados en una Institución Educativa, ubicada en la Ciudad de Cuenca, cuya población fue los estudiantes de primero de bachillerato, de los cuatro paralelos de este nivel se consideró solo uno, siendo la muestra aleatoria estratificada de 22 estudiantes y mediante un grupo testigo conformado por un docente más el investigador, se obtuvo la muestra tomada de 44 datos.

Para la recolección de información se consideró una entrevista, que según (DíazBravo et al. 2013), cuando ésta es semiestructurada puede recopilar más información porque le permite al entrevistador pedir detalles o aclarar dudas que surjan en el 
momento, en este caso con respecto a la didáctica de la matemática, además, se aplicó la técnica de observación que según (Matos \& Pasek, 2008), permite obtener información confiable de los comportamientos o conductas que tienen las personas 0 estudiantes durante la clase.

Los instrumentos para la recolección de datos fueron validados mediante el método delfi y la ficha de observación también fue verificada a través del método Alfa de Cronbach con un 0.964. Para la información proporcionada por la ficha de observación se realizó un análisis estadístico descriptivo para identificar la normalidad de las variables, tablas de frecuencias y relación de variables mediante Chi-cuadrado con el software SPSS.

\section{RESULTADOS}

Los resultados cualitativos fueron obtenidos mediante la aplicación de una entrevista a un docente licenciado en física y matemática con respecto a la didáctica de la asignatura, mientras que los cuantitativos se adquirieron a través de la ficha de observación que se aplicó en una hora de clase y fueron procesados en SPSS, con la finalidad de analizar de que manera Logic Circuit Simulator puede aportar en el aprendizaje de lógica proposicional en estudiantes de primero de bachillerato.

Respecto a la entrevista, se encontró varios puntos clave que permiten mejorar el proceso de enseñanza como indican en la tabla 4 , algunos de ellos son el dominio que el docente tiene sobre el tema a impartir, de ésta forma se puede relacionar adecuadamente contenidos teóricos con la práctica, también, la planificación adecuada que involucre y motive al estudiante con las actividades, además de, permitirles trabajar de manera cooperativa y cautivar su interés. 


\section{Tabla 4.}

Entrevista a un docente de Matemática con experiencia.

\begin{tabular}{|c|c|c|}
\hline $\begin{array}{l}\text { Unidad de } \\
\text { análisis }\end{array}$ & Categorías & Segmento \\
\hline $\begin{array}{l}\text { Proceso de } \\
\text { enseñanza }\end{array}$ & $\begin{array}{l}\text { Didáctica de la } \\
\text { Matemática }\end{array}$ & $\begin{array}{l}\text { Dominio y conocimiento de la materia. } \\
\text { Actividades lúdicas. } \\
\text { Mantener la participación activa de los estudiantes. } \\
\text { Relacionar la teória con la practicidad de los } \\
\text { contenidos. } \\
\text { Evaluación continua. }\end{array}$ \\
\hline $\begin{array}{l}\text { Razonamiento } \\
\text { lógico }\end{array}$ & Lógica Proposicional & $\begin{array}{l}\text { Fortalece el razonamiento y toma de decisiones. } \\
\text { Aplicaciones numéricas, verbales, programación, } \\
\text { electrónica, etc. }\end{array}$ \\
\hline
\end{tabular}

Elaboración: Los autores.

En cuanto a los datos cuantitativos se utilizó la estadística descriptiva, para lo cual primero se ejecutó las pruebas de normalidad, considerando el método Shapiro Wilk se obtuvo un h0 $<0.05$ para las diez variables consideradas, concluyendo que fueron de tipo paramétrico.

En la tabla 5 se analizó mediante frecuencias la variable del entusiasmo demostrado por los estudiantes mientras realizaban las diversas actividades utilizando el simulador de circuitos, se obtuvó que el $77.3 \%$ de los estudiantes mostraron entusiasmo con la temática aplicada con Logic Circuit Simulator Pro, lo cual evidencia que el aprendizaje con estrategias didácticas y uso de las TIC permite motivar e involucrar activamente al estudiante en su proceso de aprendizaje. 


\section{Tabla 5.}

El estudiante muestra entusiasmo mientras realiza las actividades con Logic Circuit Simulator Pro.

\begin{tabular}{llcccc}
\hline & Frecuencia & Porcentaje & $\begin{array}{c}\text { Porcentaje } \\
\text { válido }\end{array}$ & $\begin{array}{c}\text { Porcentaje } \\
\text { acumulado }\end{array}$ \\
\hline Válidos & Muy frecuente & 21 & 47,7 & 47,7 & 47,7 \\
& Frecuente & 13 & 29,5 & 29,5 & 77,3 \\
& A veces & 5 & 11,4 & 11,4 & 88,6 \\
& Casi nunca & 4 & 9,1 & 9,1 & 97,7 \\
& Nunca & 1 & 2,3 & 2,3 & 100,0 \\
\hline Total & 44 & 100,0 & 100,0 & \\
\hline
\end{tabular}

Elaboración: Los autores.

Posteriormente y considerando los resultados anteriores, con la técnica de chi cuadrado se buscó la relación entre la variable del entusiasmo que mostraron los estudiantes con el dominio de la temática para construir la solución de las actividades, donde se obtuvo una significación asintótica bilateral de 0.00 , valor que resulta menor que 0.05 , con lo cual se asume que las variables si están asociadas, esto puede verificarse en la tabla 6 . Esto demostró que la motivación de los estudiantes influye directamente en su interés por resolver retos de aprendizaje. 


\section{Tabla 6.}

Tabla de contingencia: Evidencia dominio de la temática para construir la solución * Muestra entusiasmo mientras realiza las actividades.

\begin{tabular}{|c|c|c|c|c|c|c|c|}
\hline & & \multicolumn{5}{|c|}{ Muestra entusiasmo mientras realiza las actividades } & \multirow[b]{3}{*}{ Total } \\
\hline & & \multicolumn{3}{|c|}{ Casi } & \multicolumn{2}{|r|}{ Muy } & \\
\hline & & Nunca & nunca & A veces & Frecuente & frecuente & \\
\hline $\begin{array}{l}\text { Evidencia dominio } \\
\text { de la temática para }\end{array}$ & $\begin{array}{l}\text { Muy } \\
\text { frecuente }\end{array}$ & 0 & 0 & 0 & 2 & 16 & 18 \\
\hline \multirow[t]{4}{*}{ construir la solución } & Frecuente & 0 & 1 & 0 & 9 & 4 & 14 \\
\hline & A veces & 0 & 1 & 5 & 2 & 1 & 9 \\
\hline & Casi nunca & 0 & 2 & 0 & 0 & 0 & 2 \\
\hline & Nunca & 1 & 0 & 0 & 0 & 0 & 1 \\
\hline Total & & 1 & 4 & 5 & 13 & 21 & 44 \\
\hline \multicolumn{8}{|c|}{ Pruebas de Chi-cuadrado } \\
\hline & & \multicolumn{2}{|r|}{ Valor } & \multicolumn{2}{|r|}{ gl } & \multicolumn{2}{|c|}{$\begin{array}{l}\text { Sig. asintótica } \\
\text { (bilateral) }\end{array}$} \\
\hline \multicolumn{2}{|c|}{ Chi-cuadrado de Pearson } & \multicolumn{2}{|r|}{$102,718^{a}$} & \multicolumn{2}{|r|}{16} & \multicolumn{2}{|c|}{0,000} \\
\hline \multicolumn{2}{|c|}{ Razón de verosimilitudes } & \multicolumn{2}{|r|}{54,771} & \multicolumn{2}{|r|}{16} & \multicolumn{2}{|c|}{0,000} \\
\hline \multicolumn{2}{|c|}{ Asociación lineal por lineal } & \multicolumn{2}{|r|}{29,192} & \multicolumn{2}{|r|}{1} & \multicolumn{2}{|c|}{0,000} \\
\hline \multicolumn{2}{|l|}{$\mathrm{N}$ de casos válidos } & \multicolumn{2}{|r|}{44} & & & & \\
\hline
\end{tabular}

Elaboración: Los autores.

En base a la información que se obtuvo, se pudo contrastar con lo que presenta (Rubio et al. 2020), dónde la lógica proposicional se trabajó mediante una base de programación y robótica, obteniendo resultados favorables a nivel cognitivo y emocional de los estudiantes, en este caso, también se evidenció que la electrónica digital básica resultó de gran interés para el alumno, permitiendole adquirir los conocimientos y aplicarlos en distintos contextos. 


\section{PROPUESTA}

Los elementos que conforman la propuesta para la enseñanza de lógica proposicional mediante herramientas digitales o circuitos lógicos reales se basa en un análisis del contexto de los estudiantes y la preparación del docente que influyen directamente en la elaboración del material didáctico y construcción de circuitos lógicos, puede observarse en la figura 1.

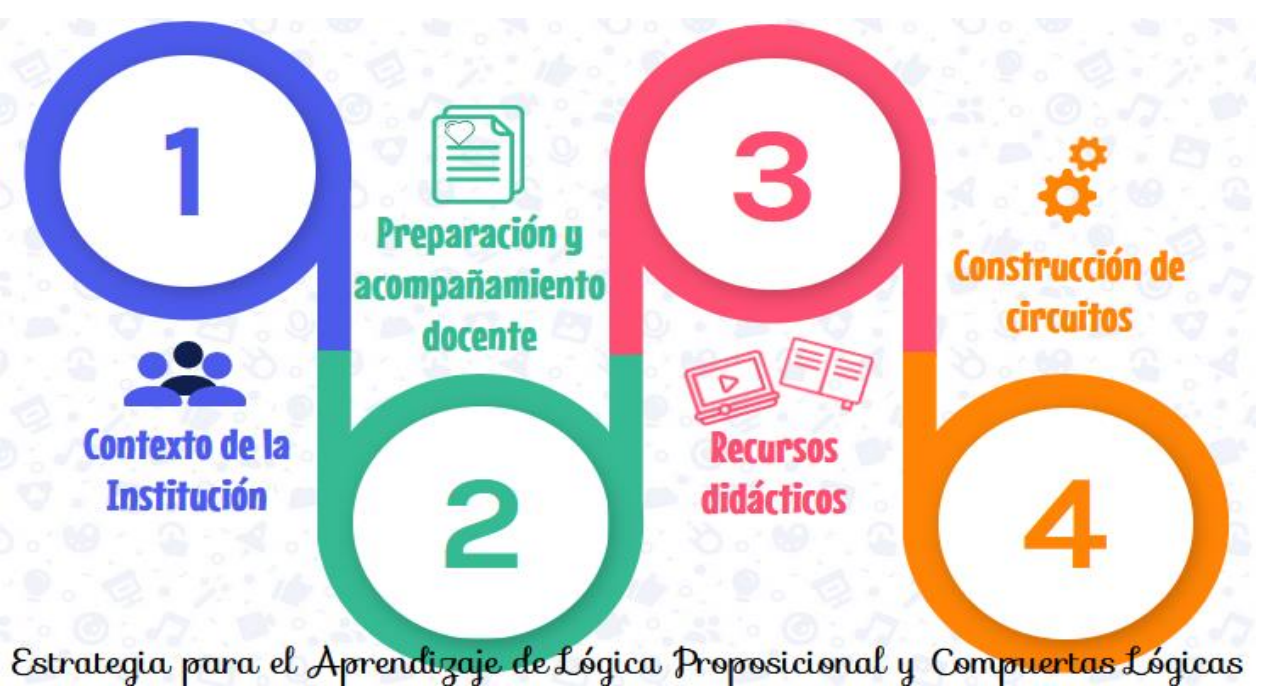

Figura 1. Propuesta EALC.

Elaboración: Los autores.

Contexto de la institución: se requiere analizar el entorno de los estudiantes, su accesibilidad a dispositivos electrónicos y manejo de los mismos.

Preparación y acompañamiento docente: el docente debe dominar los contenidos más allá de la teoría, de esta manera podrá llevarlos a prácticas reales, además de brindar un seguimiento adecuado a cada estudiante, realizar retroalimentación y evaluación continua.

Recursos didácticos: involucra el material que se debe proporcionar a los estudiantes para revisión autónoma en sus hogares sobre la temática de lógica proposicional y los recursos que utilizará en clase como guía para relacionar con 
compuertas lógicas y determinar el valor de verdad de diversas expresiones mediante la práctica.

Construcción de circuitos: mediante simuladores para dispositivos móviles como Logic Circuit Simulator Pro, Digital Circuit Simulator, entre otras apps disponibles en las tiendas del dispositivo, y para el caso de navegador puede usarse la versión demo de logica.ly o LogicSim. Dependiendo de la herramienta seleccionada, preparar la construcción del circuito para cada compuerta lógica, relacionando las entradas con interruptores o botones y la salida con luces. Luego de comprobar cada compuerta, formar expresiones lógicas para que los estudiantes estructuren la tabla de verdad y comprueben. Se pueden plantear retos donde las condiciones de las entradas provoquen una respuesta específica para que los estudiantes traten de solventarlo.

\section{CONCLUSIONES}

Los contenidos matemáticos suelen ser poco motivantes para los estudiantes, es aquí donde surge la necesidad de cambiar las propuestas dentro del aula de clase y utilizar las compertas lógicas para fortalecer la comprensión de temas y lograr llevarlos hacia aplicaciones reales, sin olvidar el contenido teórico que es fundamental para entender cómo funciona la toma de decisiones por parte de los dispositivos tecnológicos, aplicaciones digitales y en general, la vida diaria.

Para preparar un tema, la comunicación eficaz con docentes que logran llevar a sus estudiantes a mejorar su razonamiento, favorece para la elaboración de estrategias didácticas innovadoras, las cuales al ser aplicadas durante las clases se puede comprobar su efectividad mediante la participación, cuestionamientos, interés y motivación que demuestren los estudiantes en el desarrollo de las diversas actividades que son guiadas por el docente.

Es así como, estructurar una secuencia didáctica que incluya el uso de un simulador de compuertas lógicas para la enseñanza de lógica proposicional y aplicarla dentro del aula, motiva a los estudiantes a participar con preguntas que les ayude a comprender el tema y utilizarlo por ellos solos en la búsqueda de la solución a las 
distintas actividades o retos que relacionan los contenidos teóricos de lógica con la parte práctica que es el armado de circuitos lógicos.

\section{FINANCIAMIENTO}

No monetario

\section{AGRADECIMIENTOS}

A la Universidad Católica de Cuenca; por impulsar el desarrollo de este proceso investigativo desde la praxis educativa.

\section{REFERENCIAS CONSULTADAS}

Aranda-Utrero, V. (2019). La verdad lógica en el fragmento proposicional de los Principia y sus implicaciones metalógicas [Logical truth in the propositional fragment of the Principia and its metalogical implications]. Andamios, 16(41), 4361.https://doi.org/10.29092/uacm.v16i41.714

Carranza-Cortés, J. (2018). Aplicación de las tecnologías de la información y la comunicación para la búsqueda de información científica en el posgrado de especialidades médicas [Application of information and communication technologies for the search of scientific information in the graduate of medical specialties]. Anestesia en México, 30(1), 18-25.

Coronel-Maji, F., Guilcapi-Mosquera, J., \& Vargas-Guambo, J. (2018). Uso de Geogebra y su incidencia en el proceso Enseñanza- Aprendizaje de grafica de funciones en el Nivel Superior [Use of Geogebra and its incidence in the TeachingLearning process of graphs of functions at the Higher Level]. European Scientific Journal, ESJ, 14(21), 1. https://doi.org/10.19044/esj.2018.v14n21p1

Díaz-Bravo, L., Torruco-García, U., Martínez-Hernández, M., \& Varela-Ruíz, M. (2013). La entrevista, recurso flexible y dinámico [The interview: a flexible and dynamic resource]. Investigación en educación médica, 2(7), 162-167.

González-Vidal, I. (2020). Influencia de las TIC en el rendimiento escolar de estudiantes vulnerables [Influence of ICT on the school performance of vulnerable students]. RIED. Revista Iberoamericana de Educación a Distancia, 24(1), 351. https://doi.org/10.5944/ried.24.1.27960 
Hernández-Sampieri, R., Fernández-Collado, C., \& Baptista-Lucio, M. (2014). Metodología de la Investigación [Investigation Methodology] (Sexta). McGRAWHILL.

INEVAL. (2020). Infome de resultados Nacional, Examen de grado, Año lectivo 20192020. [National results report, Grade test, 2019-2020 school year]. https://n9.cl/1bofm

Lee, S., \& Park, C. H. (2019). Conductivity, superhydrophobicity and mechanical properties of cotton fabric treated with polypyrrole by in-situ polymerization using the binary oxidants ammonium Peroxodisulfate and ferric chloride. Textile Research Journal, 89(12), 2376-2394. https://doi.org/10.1177/0040517518792732

Li, F., \& Xi, Q. (2021). Research and implementation of a fabric printing detection system based on a field programmable gate array and deep neural network. Textile Research Journal. https://doi.org/10.1177/00405175211048156

Li, F., \& Xi, Q. (2021). Research and implementation of a fabric printing detection system based on a field programmable gate array and deep neural network. Textile Research Journal. https://doi.org/10.1177/00405175211048156

Lopez-Caudana, E., Ramirez-Montoya, M., Martínez-Pérez, S., \& Rodríguez-Abitia, G. (2020). Using Robotics to Enhance Active Learning in Mathematics: A MultiScenario Study [Uso de la robótica para mejorar el aprendizaje activo en matemáticas: un estudio de múltiples escenarios]. Mathematics, 8(2163). https://doi.org/10.3390/math8122163

Matos, Y., \& Pasek, E. (2008). La observación, discusión y demostración: técnicas de investigación en el aula [The observation, discussion and demonstration: technical of investigation in the classroom]. Laurus, 14(27), 33-52. https://n9.cl/93je2

Mercader-Ruiz, J., Presentación-Herrero, M. J., \& Siegenthaler-Hierro, R. (2017). Influencia De Las Habilidades Matemáticas Básicas En El Rendimiento Posterior [Influence of early numeracy skills on later performance]. International Journal of Developmental and Educational Psychology. Revista INFAD de Psicología., 3(1), 243. https://doi.org/10.17060/ijodaep.2017.n1.v3.993

MINEDUC. (2016). Educación Obligatoria Nivel Bachillerato, Tomo 2 [Obligatory Education, High school level, Volume 2]. https://n9.cl/1frin 
Rincón-Rueda, A., \& Ávila-Díaz, W. (2016). Una aproximación desde la lógica de la educación al pensamiento computacional [An approach from the logic of the education to the computational thinking]. Sophía, Colección de Filosofía de La Educación, 2(21), 161. https://doi.org/10.17163/soph.n21.2016.07

Rodríguez-Gómez, D., Castro, D., \& Meneses, J. (2018). Usos problemáticos de las TIC entre jóvenes en su vida personal y escolar [Problematic uses of ICTs among young people in their personal and school life]. Comunicar: Revista Científica Iberoamericana de Comunicación y Educación, 56, 91-100. https://doi.org/10.3916/C56-2018-09

Rubio, G., Guaraca, P., \& Amaya, P. (2020). Desarrollo del pensamiento computacional: robots educativos en el ambiente de aprendizaje de robótica en Educación Inicial [Development of computational thinking: educational robots in the robotics learning environment in Early Childhood Education]. Mamakuna, (14), $24-42$.

UNESCO. (2013). Enfoques estratégicos sobre las TICS en educación en América Latina y el Caribe [Strategic approaches on the use of ICTS in education in Latin America and the Caribbean]. https://n9.cl/lngoh

Vega-Vega, J., Niño-Duarte, F., \& Cárdenas, Y. (2015). Enseñanza de las matemáticas básicas en un entorno e-Learning: un estudio de caso de la Universidad Manuela Beltrán Virtual [Teaching basic mathematics in an eLearning environment: a case study from the Manuela Beltrán Virtual University]. Revista EAN, O(79), 172-185. https://n9.cl/s3ch0

Zhang, J., Wu, J., Hu, X., \& Zhang, X. (2020). Multi-color measurement of printed fabric using the hyperspectral imaging system. Textile Research Journal, 90(9-10), 1024-1037. https://doi.org/10.1177/0040517519883953

Zumaeta-Arista, S., Fuster-Guillen, D., \& Ocaña-Fernández, Y. (2018). El afecto pedagógico en la didáctica de la matemática - Región Amazonas desde la mirada fenomenológica [Pedagogical Affection in Didactics of Mathematics - Amazonas Region from the Phenomenology Perspective]. Propósitos y Representaciones, 6(1), 409-462. https://doi.org/10.20511/PYR2018.V6N1.200 